EXTRAGALACTIC BACKGROUND RADIATION AND COSMOLOGY 


\title{
SPECTRUM AND ORIGIN OF THE EXTRAGALACTIC OPTICAL BACKGROUND RADIATION
}

\author{
J. Anthony Tyson \\ AT\&T Bell Laboratories \\ Murray Hill, NJ 07974 USA
}

\begin{abstract}
Ultra-deep CCD surveys to $29 \mathrm{mag}$ from .32 to $.9 \mu$ wavelength reveal an isotropic population of very blue galaxies. There are over 150,000 of these objects per square degree per magnitude. Saturation of their number density at $27 \mathrm{mag}$ indicates that most of the optical light from this population has been detected. The resulting extragalactic background radiation from the UV to the near-IR due to this population of objects is shown. Gravitational lens and Lyman-break observations show that the redshift of galaxies fainter than $24 \mathrm{mag}$ is in the range 1-3. Small-scale dark lane structures may be intergalactic dust clouds or tunnels through the luminous galaxy distribution.
\end{abstract}

\section{INTRODUCTION}

The extragalactic background radiation in the optical part of the spectrum originates in young stellar populations in galaxies at moderate to high redshift. The subject has a long history, but only recently have charge-coupled device (CCD) surveys achieved the required sensitivity to capture most of the extragalactic light of the night sky. These surveys have revealed a population of faint blue resolved objects (hereafter referred to as galaxies, although they may not be galaxies in the familiar sense) which cover the sky apparently isotropically. There was already evidence of a bluing trend with faintness for field galaxies in the photographic work of Kron (1980, 1982) (.2 mag of bluing in B-R from 18 to 23 B magnitude). The deep CCD surveys have revealed most of this population now, exhibiting over 2.5 mag of bluing in B-R to $28 \mathrm{~B}$ magnitude. My collaborators in this work have been primarily Pat Seitzer and Raja Guhathakurta. Software for automated photometry was developed in collaboration with John Jarvis, Frank Valdes, and Rick Wenk.

The intensity of the extragalactic background light (EBL) is influenced strongly by galaxy evolution (stellar lifetimes and population evolution), and to a much lesser extent by cosmology (Harrison, 1964). Number-magnitude counts which rise with magnitude like dex (0.4 $\mathrm{mag}$ ) or faster produce a brighter EBL, and if this slope never falls below 0.4 the surface brightness of the night sky would diverge. These considerations are related to Olbers's paradox. The finite luminous lifetime of stellar populations offers a way out of this divergence.

\section{THEORETICAL ESTIMATES}

Whitrow and Yallop (1965) first gave the form of the bolometric flux in an arbitrary cosmology with coeval galaxies starting at some formation redshift. Tinsley $(1977,1978,1980,1981)$ pioneered theoretical estimates of galaxy evolution. The optical EBL is probably due to evolving spirals: Wyse (1985) estimates that less than $18 \%$ of the EBL light is from ellipticals, and that the elliptical light is red. Yoshii and Takahara (1988) estimated a theoretical no-evolution EBL flux of $3 \times 10^{-6} \mathrm{erg} \mathrm{cm}^{-2} \mathrm{~s}^{-1} \mathrm{sr}^{-1} \mu^{-1}$ at $.45 \mu$ wavelength. For evolving galaxies with 
formation redshifts ranging from 3 to 5 (and nearly independent of deceleration parameter), they estimate an EBL about two times higher. EBL from faint galaxies is very sensitive to evolution and relatively insensitive to cosmology, particularly at wavelengths where much of the redshifted UV from early star formation appears. Differential galaxy counts in the blue part of the spectrum are relatively more sensitive to evolution. We have run various models, using different evolution scenarios (including various stellar burning histories and merging), galaxy luminosity functions, stellar initial mass functions, and cosmological parameters. We find that little if any cosmological information can be uniquely determined from the numbermagnitude counts. What is needed is redshift information. The sum total EBL, being an integral over the flux times number counts at each wave band, is even less sensitive to cosmology. Generally, small changes in the galaxy luminosity function can compensate for changes in $q_{o}$.

\section{UPPER LIMITS TO THE OPTICAL EXTRAGALACTIC BACKGROUND}

For sufficiently strong source luminosity evolution, the diffuse background light can be measurably enhanced. Although properties of this additional diffuse background light can be indicative of galaxy evolution, the counts of faint resolved galaxies are more sensitive to evolution, because this diffuse background is an integral over the counts. Nevertheless it is useful to derive this diffuse background value for comparison with other observations of unresolved background light at various wavelengths. Upper limits to the EBL have been set by all-sky photometry (Roach and Smith, 1968; Mattila, 1976; Dube et al. 1977, 1979). Progress in this field was reviewed by Toller (1983). A convenient but unfortunate unit often used to express the light of the night sky is $S_{10}(\lambda)$, which is the equivalent bolometric surface brightness of a 10 th mag star of specified spectral type measured at wavelength $\lambda$, if its light is uniformly spread over a square degree of sky. Since measurements are always made at some wavelength, and the spectrum of the EBL is not known a priori, a better unit would be $F_{\lambda}$, the flux density at wavelength $\lambda$ in units of erg $\mathrm{cm}^{-2} \mathrm{~s}^{-1} \AA^{-1} \mathrm{sr}^{-1}$. An even more physical unit is $\lambda F_{\lambda}=v F_{v}$ in $\mathrm{W} \mathrm{m}^{-2} \mathrm{sr}^{-1}$, which gives equal weight to the energy contribution at each wavelength.

All sky photometry has yielded upper limits around $4 S_{10}(V)$, where $1 S_{10}(V)=1.2 \mathrm{x}$ $10^{-9} \mathrm{erg} \mathrm{cm}{ }^{-2} \mathrm{~s}^{-1} \mathrm{sr}^{-1} \AA^{-1}=5.3 \times 10^{-9} \mathrm{~W} \mathrm{~m}^{-2} \mathrm{sr}^{-1}$, at $4400 \AA$. Dube et al. (1979) observed an $\mathrm{EBL}$ at $5115 \AA$ of $1 \pm 1.2 S_{10}(5115 \AA)$. If the objects dominating the EBL appear smaller than $5 "$, as the data imply, then our direct imaging sensitivity is over 100 times the sensitivity of the integrated sky chopping techniques.

Gunn (1965) and Shectman $(1973,1974)$ considered statistically the fluctuations in the EBL due to galaxy clustering. This is directly measurable in our data, and this will be reported elsewhere. Partridge and Peebles (1967) estimated the light from primeval galaxies in a model in which star formation began early (high redshift), giving the EBL an IR excess in our observed frame. Our data suggest a blue color for the EBL associated with resolved objects less than about 60" in size. Since we detect and photometer discrete objects, we are not sensitive to any uniform component in the EBL, so that the EBL discussed here must be considered a lower limit if there were a smooth extragalactic visible component uniform on greater than $1^{\prime}$ scales. Recent CCD chopping observations (Guhathakurta and Tyson 1989) over angles of 20' have failed to detect any objects larger than the observed arcsec-scale galaxies, to comparable surface brightness. Thus the angular size distribution would have to be bimodal over more than three decades in angle (1-1000") if there were any isotropic flat featureless EBL component of comparable surface brightness. 


\section{DIRECT MEASUREMENT OF THE OPTICAL EBL FROM DISCRETE OBJECTS}

Providing the observations go to sufficiently faint surface brightness to catch all the light from these objects, the EBL from all discrete objects can be calculated directly from our galaxy count data. Deep imaging in several wavelength bands can be used to construct a rough spectral distribution for this EBL. This may then be compared with the limits from other techniques and with theories for various scenarios for galaxy evolution.

\subsection{CCD Surveys}

Deep CCD imaging surveys have been carried out in 13 random high galactic latitude fields to $29 B_{J}, 28 \mathrm{R}$, and $26 \mathrm{I} \mathrm{mag} \operatorname{arcsec}^{-2}$ limiting surface brightness (Tyson 1988; Tyson and Seitzer 1988). Number-magnitude counts and accurate photometry were obtained for over 7000 faint galaxies. Recently, ultra-deep CCD imaging to $30 \mathrm{mag} \operatorname{arcsec}^{-2}$ has been obtained in three fields (Guhathakurta, Tyson, and Majewski 1989). These surveys involve as many as 100 disregistered exposures of one field in a given filter. This 3-d stack of disregistered exposures contains all the information about the systematic errors in the CCD, telescope and sky, and the true object luminosity distribution, in separable form. Data processing (see Tyson and Seitzer 1988 and references therein) produces a catalog of object multi-band photometric data.

\subsection{Number-Magnitude Counts vs. Wavelength}

The $N(\mathrm{~m})$ counts in the $B_{J}$ band has average slope 0.45 . Note that this slope of 0.45 is supercritical in the sense that the contribution to the EBL is monotonically increasing with magnitude. The $N(m)$ slope in the U-band is even steeper. The slope of the $N(m)$ counts for longer wavelengths is sub-critical, going to a "no-evolution" slope of .3 at $.9 \mu$ wavelength. The enhancement of the blue and $U$ band counts is apparently coming from UV-bright objects, in their rest frame. Tinsley $(1977,1978,1980,1981)$ was the first to point out that star formation at a given epoch would produce a bump in the number-magnitude counts. The small but highly significant bump seen in our counts around $25 B_{J}$ mag is very likely this predicted effect. Is it possible that we are seeing most of the star formation in these galaxies?

The leveling off of the $N(m)$ counts at 27 B mag is now known to be real: the recent ultra-deep images of the same fields show few new galaxies. In particular, there are occasional patches relatively devoid of galaxies in the original deep CCD survey which do not fill in with new faint galaxies in the ultra-deep images in the $\mathrm{U}, B_{J}$, and $\mathrm{R}$ bands. The sum of all the imaging data for one survey field (south galactic pole) is shown in Figure 1, in which the limiting apparent magnitude is about 30th mag. Patches of low galaxy count do not appear to fill in with faint galaxies as fainter limiting magnitudes are reached.

\subsection{EBL from Integral Flux Counts}

Galaxy counts vs isophotal magnitude may be used to calculate directly the EBL, down to the surface brightness threshold for detection. Our CCD data, covering the range .3-1 $\mu$, can be used to calculate the EBL down to $31 B_{J}$ mag $\operatorname{arcsec}^{-2}$. The observed counts flatten fainter than $27 B_{J}$ mag, with most of the EBL flux originating from galaxies around $24 \mathrm{~B}$ mag. Integrating the flux corresponding to our differential number-magnitude counts, bracketing the 


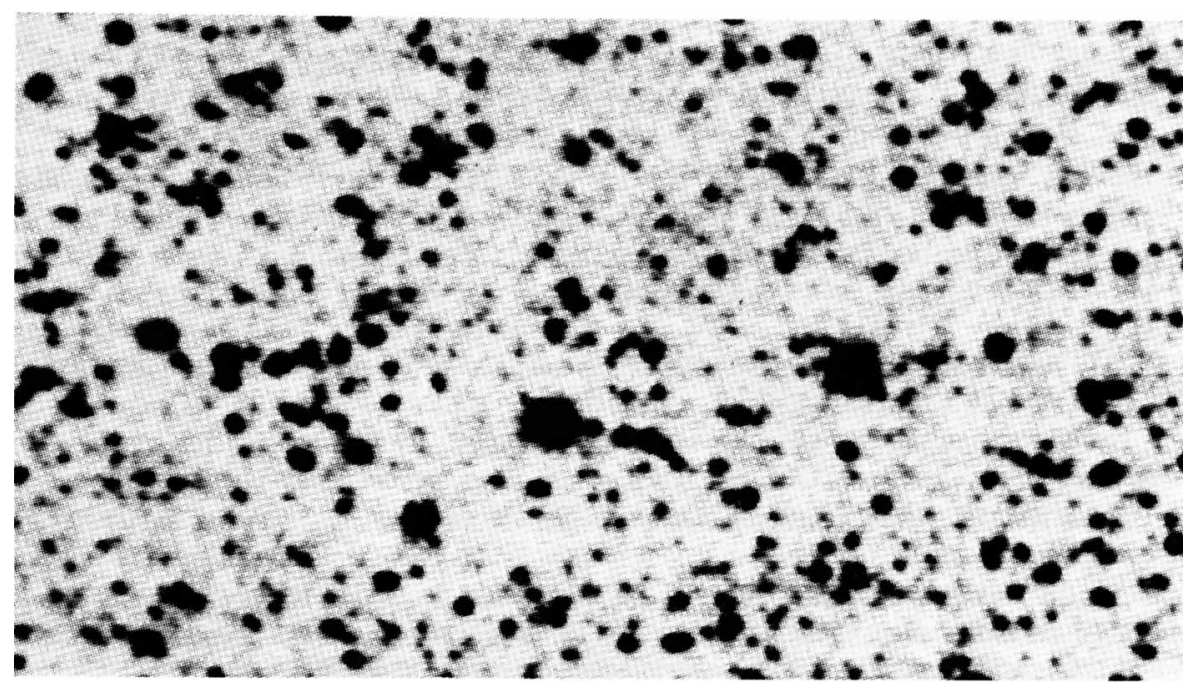

Figure 1. The sum of all $U, B_{J}, R$ images of the south galactic pole field. This is one of the three ultra-deep fields produced in the latest CCD survey. The limiting broadband magnitude in this image is about 30 mag. This is a negative: bright objects are black. The field measures $2.3 \times 4.7$ : The "dark" lanes seen on shorter exposures do not appear to fill in with galaxies. If due to dust, it must be very distant dust (otherwise it would appear bright due to reflected disk light [Guhathakurta and Tyson 1989]) with large extinction, implying a massive contribution by dust and gas to $\Omega$. If not due to dust, we may be seeing through tunnels in the galaxy topology out beyond the first shell of stellar production.

uncertainties in the counts fainter than $28 B_{J}$ mag, we arrive at an EBL at wavelengths $\lambda=3200-10000 \AA$ of

$$
\begin{aligned}
& F_{\lambda}=7.0(+.2-.1) \times 10^{-10} \mathrm{erg} \mathrm{cm}^{-2} \mathrm{~s}^{-1} \mathrm{sr}^{-1} \AA^{-1} \text { at } 3600 \AA . \\
& F_{\lambda}=6.4(+.2-.1) \times 10^{-10} \mathrm{erg} \mathrm{cm}^{-2} \mathrm{~s}^{-1} \mathrm{sr}^{-1} \AA^{-1} \text { at } 4500 \AA . \\
& F_{\lambda}=4.5(+.2-.1) \times 10^{-10} \mathrm{erg} \mathrm{cm} \mathrm{s}^{-1} \mathrm{sr}^{-1} \AA^{-1} \text { at } 6500 \AA . \\
& F_{\lambda}=2.9(+.3-.2) \times 10^{-10} \mathrm{erg} \mathrm{cm} \mathrm{s}^{-1} \mathrm{sr}^{-1} \AA^{-1} \text { at } 9000 \AA .
\end{aligned}
$$

In the blue this is 30 times the surface flux threshold of these observations and is equivalent to $0.53(+.02-.01) S_{10, G 2 V}(V)$. The flattening of the $\mathrm{N}(\mathrm{m})$ counts originally seen in the $B_{J}$ band (Tyson 1988) have been seen now in the $U$ band, and thus represents the outer shell of magnitude contributing significantly to the EBL. The quoted errors bracket the remaining differential $\mathrm{N}(\mathrm{m})$ uncertainties fainter than $28 B_{J}$ mag (flat or falling), and partially from field-to-field count variations. Our accuracy is not limited due to any star/galaxy classification problems at the faint end ( $\left.>27 B_{J} \mathrm{mag}\right)$, since galaxies outnumber stars by several orders of magnitude at that point. Diffuse objects larger than 30"are not counted in the EBL sum in equation (1), but in view of recent observations any such addition to the EBL appears to be negligible. This EBL we have measured is equivalent to a diffuse surface brightness of $28.8 B_{J} \mathrm{mag} \operatorname{arcsec}^{-2}$. Galaxies fainter than $20 B_{J}$ mag contribute about $75 \%$ of the EBL at $4500 \AA$. The redshift shell which dominates the EBL is dependent on the galaxy 
luminosity evolution $\mathrm{L}(\mathrm{z})$. The corresponding magnitude shell contributing most to the EBL is that magnitude beyond which the slope $\mathrm{dN} / \mathrm{dm}$ drops below 0.4: $26 B_{J} \mathrm{mag}$. The $B_{J}-\mathrm{R}$ color of those objects is near zero, indicating that most of the energy of the .3-1 $\mu$ EBL is in the blue.

\subsection{Spectral Distribution}

Our U, $B_{J}, R$, and I surface flux integrals for this EBL are plotted in Figure 2 vs wavelength. Note the monotonic rise to the short wavelength end. The error bars on our EBL flux are $3 \sigma$, and are mostly due to field-to-field fluctuations in numbers of bright galaxies. For comparison, a G-type star spectrum is also shown. Note the UV excess of the EBL. Our measured spectral distribution of the EBL is slightly redder than flat in $F_{v}$ (see Guhathakurta, Tyson, and Majewski 1989). This SED is more UV-bright than nearby galaxies and is due to evolution in this faint galaxy population.

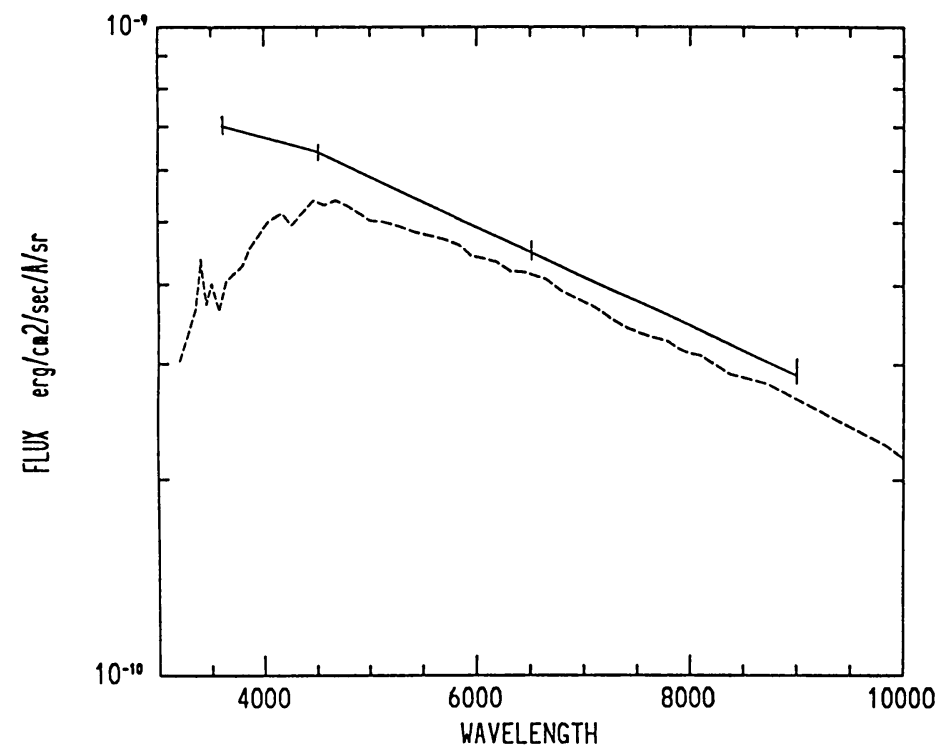

Figure 2. The sum extragalactic background flux from the faint galaxy population as a function of wavelength from $3200-10000 \AA$. The error bars are $3 \sigma$, arising mostly from field-to-field fluctuations in the brighter galaxy numbers. Adjacent wavelength (color) errors are smaller. For comparison, a Gtype stellar spectrum (typical of nearby galaxies) is plotted also. Note the blue excess of the EBL spectral energy distribution.

\subsection{Extension to the UV}

A review of non-imaging EBL surveys in the UV was given by Paresce and Jakobsen (1980). Our EBL at $450 \mathrm{~nm}$ due to this faint galaxy population is over twice the no-evolution predictions and suggests redshifts around 1-3, for a source spectrum rising in the UV to Lyman- $\alpha$. More sensitive UV and near-UV surveys could determine if the galaxy counts at a given flux level are significantly below that seen at $320-450 \mathrm{~nm}$, as might be expected in this model. If 
spiral formation continues to redshifts near one, then the new UV sensitive CCDs could be used to detect the bright galaxies expected in the $2400-3000 \AA$ band from an orbiting telescope. The Hubble Space Telescope may detect the bright nuclei of some of these blue galaxies. Galaxies fainter than 24 B mag contribute about $30 \%$ of the EBL in the blue. Since the redshift of this faint population is mainly larger than 0.9 , their expected contribution to $10^{\prime \prime}$ scale fluctuations of the EBL would be negligible for wavelengths shorter than $1700 \AA$ if these galaxies are "black" in their Lyman continuum. The rocket-borne telescope of Martin and Bowyer (1988) made an interesting measurement at $1600 \AA$ of $16-52$ photon $\mathrm{cm}^{-2} \mathrm{~s}^{-1} \mathrm{sr}^{-1}$. This corresponds to a flux of $2-6 \times 10^{-10} \mathrm{erg} \mathrm{cm}^{-2} \mathrm{~s}^{-1} \AA^{-1} \mathrm{sr}^{-1}$, or $0.3-1 \mathrm{nW} \mathrm{m} \mathrm{sr}^{-1}$. It would be useful to have a direct imaging UV observational test.

\subsection{Extension to the IR}

Is the sky similarly crowded with faint galaxies at $\mathrm{K}$ band? The current generation of InSb 2micron imagers are almost sensitive enough to capture much of the light from this population of galaxies, but they are nonlinear and difficult to calibrate at surface brightnesses which are a small fraction of the sky background. The new $\mathrm{HgCdTe}$ area imagers will surely cause as much of a revolution in K-band imaging as the silicon CCDs have in the optical. Modeldependent estimates of the $\mathrm{K}$-band galaxy counts can be attempted by fitting a range of models to our $0.3-1 \mu$ counts and then extrapolating to $2.2 \mu$. Realizing that this is a dangerous procedure, since galaxy evolution models currently involve arbitrary scenarios, I take as an example one evolution model (Tyson 1988) which fits our optical counts well.

The integrated K-band EBL, assuming no dust and no star formation at redshifts greater than 6, estimated from the extrapolated model counts is

$$
1.4 \pm .4 \times 10^{-10} \mathrm{erg} \mathrm{cm}^{-2} \mathrm{~s}^{-1} \AA^{-1} \mathrm{sr}^{-1} \text { at } 2.2 \mu .
$$

This must be taken as a lower limit, since it involves no early stellar formation $(z>6)$ nor hot dust. It is conceivable that there was some early stellar burning at redshifts higher than 6 (so as to avoid enhancing our $.9 \mu$ galaxy counts) substantially raising the $\mathrm{K}$-band counts and total flux. If there were a bright phase of stellar burning at redshift about 10-20 (population 3 stars), they would be seen in a deep K-band survey. Accurate imaging of these galaxies to low surface brightness in K-band will be revealing. Boughn et al. (1986) obtained an upper limit of $7 \times 10^{-20} \mathrm{erg} \mathrm{cm}^{-2} \mathrm{~Hz}^{-1} \mathrm{sr}^{-1}=4 \times 10^{-10} \mathrm{erg} \mathrm{cm}^{-2} \mathrm{~s}^{-1} \AA^{-1} \mathrm{sr}^{-1}$ at $2.2 \mu$ for fluctuations on scales of 10-30", just a factor of 3 above our predicted EBL at K-band.

\subsection{Isotropy}

The faint galaxy counts in 12 random high galactic latitude fields are isotropic on the angular scales between fields $\left(10-180^{\circ}\right)$ to about $10 \%(2 \sigma)$. This is shown in Figure 3. Since these galaxies are at redshifts of order 1 and above, the galaxies in different fields are out of causal communication (reminiscent of the cosmic microwave background). On angular scales smaller than $30^{\prime \prime}$ we find reproducible structures in each of our survey fields (see Figure 1). Whether these "dark lanes" are intergalactic dust clouds (with implications for $\boldsymbol{\Omega}_{\text {baryon }}$ ) or open tunnels in the galaxy distribution is under investigation. 


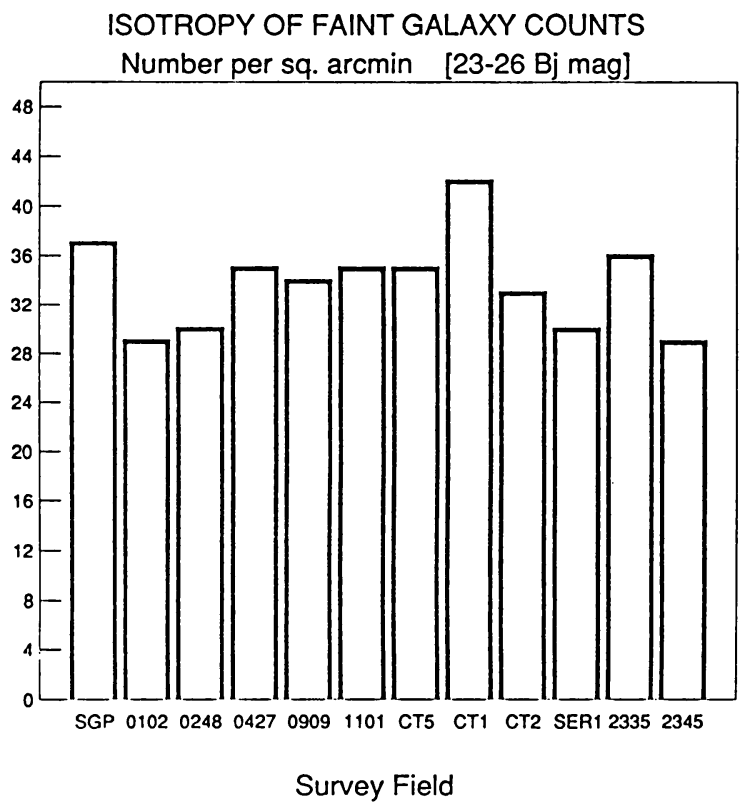

Figure 3. The distribution of the faint galaxy counts on the sky. The 12 survey fields are all at high galactic latitude, and show about twice the fluctuation expected from a random distribution. Thus, this population is isotropic to $2 \sigma$ even though the fields are out of causal communication.

\section{ORIGIN OF THE OPTICAL EBL}

The spectral distribution of the EBL, in $\lambda F_{\lambda}=v F_{v}$ units, is shown in Figure 4. Speculation on the nature of these faint blue galaxies has ranged from low redshift dwarf galaxies undergoing star formation, to high redshift primeval galaxies forming the majority of their initial stellar population. Although the differential number-magnitude counts will be helpful in constraining theories of the nature of these objects, number-redshift information will be much more effective in narrowing the field of candidates. Although detailed spectroscopy at $10 \AA$ resolution would be desirable, these 26-28 mag galaxies are too faint. Redshift constraints on this faint population must be obtained in other ways.

\subsection{Upper Redshift Limit}

An upper redshift limit for this population of faint blue galaxies is immediately apparent from its spectral energy distribution shown in Figure 2. If a significant fraction of these galaxies were at redshift greater than 2.8 , the Lyman break would be shifted through the U passband, causing these galaxies to either drop out in the $U$ band or to have a large drop in flux between $B_{J}$ and U. Lyman breaks from most stars in these primeval galaxies are probably at least a factor of 2. In addition, these galaxies would have even more hydrogen than present galaxies, which would absorb all the Lyman continuum photons, causing these galaxies to be black in the U-band for redshifts larger than 3 (Guhathakurta, Tyson, and Majewski 1989). 


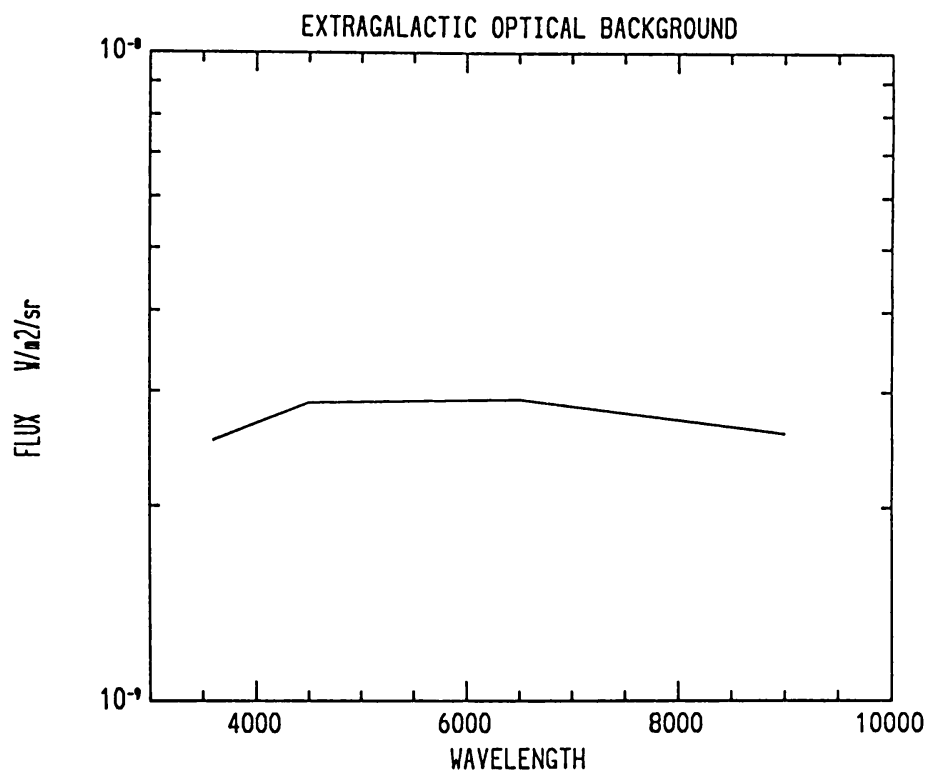

Figure 4. The extragalactic background radiation spectral energy distribution in $\lambda F_{\lambda}=v F_{\mathrm{v}}$ mks units ( $\mathrm{W} \mathrm{m} \mathrm{m}^{-2} \mathrm{sr}^{-1}$ ) over optical wavelengths.

\subsection{Lower Redshift Limit}

A lower redshift limit for this faint population can be derived from its response to a gravitational lens placed in front of it. A good test is provided by the dark matter lens in the cluster $1409+52$ at a redshift of 0.46 . The same number of faint blue galaxies per square arcminute are seen behind this red cluster, but most of their images are stretched by the gravitational lens and are aligned orthogonal to the vector to the cluster center (Tyson, Valdes, and Wenk 1989). The distribution of alignments of the blue galaxies contrasts with that of the red cluster galaxies: there is an excess in the tangent alignment bin for faint blue galaxies. This can only occur if more than $70 \%$ of these galaxies have redshifts greater than 0.9 .

\subsection{Protogalaxies}

Various models of primeval galaxies predict extended low surface brightness morphology. We find no consistent evidence for the expected numbers of these extended objects in the conservatively-thresholded FOCAS catalogs. However, their surface brightness may be below $31 B_{J} \mathrm{mag} \operatorname{arcsec}^{-2}$. In the absence of significant dust, it is possible that many primevals would be mixed with the large numbers of faint blue galaxies. In this connection, it is perhaps significant that we do not see many extended very red (in R-I) objects at faint I magnitudes. Either there was negligible galaxy formation (proto-ellipticals and proto-bulges) for $z>5$, or there was adequate dust from a very much earlier star formation epoch to dim that luminous phase below detection. Ostriker and Heisler (1984) argue that dust obscuration could become severe at redshifts $3-4$. 
The apparent optical counterpart of 3C326.1 (McCarthy et al., 1987) is a primeval galaxy candidate. Most primevals are expected to be at least 2-3 magnitudes fainter than this, otherwise they would have been found in emission line surveys (Koo and Kron, 1980; Koo, 1986; Pritchet and Hartwick 1987). In their hierarchical clustering model, Baron and White (1987) find that 60 percent of the stars in a galaxy form before collapse of the initial galaxy-scale perturbation. If, as our data suggest, early star formation in galaxies is continuous and spread out over at least $10^{9}$ years, earliest galaxy formation redshifts can be high (say 5-10) but much star formation may be ongoing at a redshift of 2 or lower.

All current theories of galaxy formation (e.g., Davis et al., 1985) predict a high degree of clumping for primevals at moderate to low redshift. Unfortunately, such clumps of low surface brightness galaxies could appear as a single diffuse "protogalaxy." Recently, Baron and White (1987) have made numerical simulations of inhomogeneous galaxy formation in the CDM model. They predict that primevals will be more difficult to identify spectroscopically than was previously thought. However, they should be observable in this deep CCD survey, and their low-level surface brightness morphology is within reach of present $\mathrm{CCD} /$ telescope technology. It is possible that we are seeing the first epoch of star formation in these galaxies, and in that sense these blue galaxies would be primevals.

\subsection{Morphology of Faint Blue Galaxies}

After deconvolution (Tyson and Wenk 1990), galaxies in our sample fainter than $26 B_{J}$ mag are found to have average exponential scale length $0.2-0.3$ ". Over the magnitude range of 17 to $27 B_{J}$ mag of our CCD sample, there is no evidence for a significant tail past 3" radius in the galaxies, and all but two of the faint galaxies with central surface brightnesses fainter than $24 B_{J}$ mag $\operatorname{arcsec}^{-2}$ have scale lengths less than 1". Galaxies of 26th $B_{J}$ mag appear to be no more extended in comoving metric size than brighter galaxies at low redshift. From the model experiments described above, this evidence favors bulge+disk evolution models at redshifts larger than 1.5 , for low $\Omega$. To $29 B_{J} \mathrm{mag} \operatorname{arcsec}^{-2}$, there is no evidence of bimodality in the angular size distribution of galaxies.

The blue galaxies in our deep sample have considerably higher ellipticities than those in clusters. Only about $10 \%$ of the faint galaxies have observed (uncorrected) ellipticities less than 0.2 . When corrected for seeing, the $a / b$ ratios will increase even further. Comparison of this observed flattening with the nearby population of galaxies is in progress. We may be seeing spiral galaxy disks in formation.

\section{SUMMARY}

We find an isotropically distributed population of UV-excess galaxies in a redshift range 1-3. Their number density is high: over $150,000 \mathrm{deg}^{-2} \mathrm{mag}^{-1}$. There appears to be a leveling off of the $N(m)$ counts in the blue and $U$ wavelength bands, giving most of the contribution to the extragalactic background light at magnitudes between 24 and $26 \mathrm{U}$ mag.

Aknowledgments - I would like to acknowledge my collaborators in this research over the past ten years: Pat Boeshaar, Raja Guhathakurta, John Jarvis, Steve Majewski, Pat Seitzer, Frank Valdes, and Rick Wenk. 


\section{REFERENCES}

Baron, E. and White, S. D. M. 1987, Astrophys. J., 322, 585.

Boughn, S. P., Saulson, P. R., and Uson, J. 1986, Astrophys. J., 301, 17.

Davis, M., and Wilkinson, D. T. 1974, Astrophys. J., 192, 251.

Dube, R. R., Wickes, W. C., and Wilkinson, D. T. 1977, Astrophys. J., 215, L51.

Dube, R. R., Wickes, W. C., and Wilkinson, D. T. 1979, Astrophys. J., 232, 333.

Guhathakurta, P. and Tyson, J. A. 1989, Astrophys. J., in press.

Guhathakurta, P., Tyson, J. A., and Majewski, S. 1989, Astrophys. J. Lett., in press.

Gunn, J. E. 1965, Ph.D.Thesis,

Harrison, E. R. 1964, Nature, 204, 271.

Koo, D. C. 1986, Spectral Evolution of Galaxies, eds. C. Chiosi and A. Renzini (Dordrecht: Reidel), 419.

Koo, D. C. and Kron, R. G. 1980, Pub. Asron. Soc. Pacific, 92, 537.

Kron, R. G. 1980, Astrophys. J. Supp., 43, 305.

Kron, R. G. 1982, Vistas in Astronomy, $26,37$.

Martin, C. and Bowyer, S. 1989, Astrophys. J., 338, 677.

Mattila, K. 1976, Astr. Astrophys., 47, 77.

McCarthy, P. J., Spinrad, H., Djorgovski, S., Strauss, M., van Breugel, W., and Liebert, J. 1987, Astrophys. J. Lett., 319, L39.

Ostriker, J. P. and Heisler, J. 1984, Astrophys. J., 278, 1.

Paresce, F. and Jakobsen, P. 1980, Nature, 288, 119.

Partridge, R. B., and Peebles, P. J. E. 1967, Astrophys. J., 147, 868.

Partridge, R. B., and Peebles, P. J. E. 1967, Astrophys. J., 148, 377.

Pritchet, C. J. and Hartwick, F. D. A. 1987, Astrophys. J., 320, 464.

Roach, F. E. and Smith, L. L. 1968, Geophys. J. R. A. S., 15, 227.

Shectman, S. A. 1973, Astrophys. J., 179, 681.

Shectman, S. A. 1974, Astrophys. J., 188, 233.

Tinsley, B. M. 1977, Astrophys. J., 216, 349.

Tinsley, B. M. 1978, Astrophys. J., 220, 816.

Tinsley, B. M. 1980, Astrophys. J., 241, 41.

Tinsley, B. M. 1981, Astrophys. J., 250, 758.

Toller, G. N. 1983, Astrophys. J., 266, L79.

Tyson, J. A. 1988, Astrophys. J., 96, 1.

Tyson, J. A. and Seitzer, P. 1988, Astrophys. J. 335, 552.

Tyson, J. A., Valdes, F., and Wenk, R. A. 1989, Astrophys. J. Lett., in press.

Tyson, J. A. and Wenk, R. A. 1990, in preparation.

Whitrow, G. J. and Yallop, B. D. 1964, MNRAS, 127, 301.

Whitrow, G. J. and Yallop, B. D. 1965, MNRAS, 130, 31.

Yoshii, Y. and Takahara, F. 1988, Astrophys. J., 326, 1.

Wyse, R. F. G. 1985, Astrophys. J., 299, 593.

G. Verschuur: Does the turn-down in the log $N$ versus magnitude diagram imply you are seeing the edge of the universe of galaxies?

A. Tyson: Only in the sense that it would be an "apparent" edge in the optical. If there were sufficient dust to obscure galaxies beyond about redshift 3 in most directions, for example, then we might see this effect in the $\mathrm{N}(\mathrm{m})$ counts. If one could show that there is insufficient dust, however, then this decrease in the $N(m)$ slope for $B>26$ mag could indicate that we are seeing back to the earliest galaxies. In that case, the absence of any steep slope in the I-band counts pushes any epoch of earlier star formation to redshifts of greater than 7.

M. Harwit: To what do you attribute the dark lanes in the galaxy distribution? Since they persist to increasing sensitivity, does that mean you've reached the earliest galaxies?

A. Tyson: It is possible that, if any dust is optically thin, these regions which are relatively unpopulated by galaxies could result from a filamentary (nonhomogeneous) galaxy distribution. In that case, we would be looking out beyond the earliest galaxies through these "holes." The dark lanes cannot be due 
to nearby dust as is the $100 \mu \mathrm{m}$ cirrus because it would be optically thick and would appear bright and diffuse due to scattered disk light (as does the IRAS cirrus we have studied in the optical). If due to optically thick dust far from our galaxy, we would expect redder mean colours of galaxies inside these lanes. This test is in progress.

P. Biermann: Using standard evolutionary models for galaxies, you find that $\mathrm{vS}_{\mathrm{v}}$ is about the same in the far infrared as in the optical, maybe even more, if you think of M82 or other starburst galaxies. Therefore, what would you expect to see at 100-200 $\mu \mathrm{m}$ if you could do it, and what would be the contribution to the diffuse light there from all these galaxies?

A. Tyson: Our observed spectrum of the extragalactic background light from this population of faint blue galaxies is indistinguishable from that of a G-type star, for wavelengths between 5,000 and 10,000 $\AA$. Thus, unless there were considerable dust in these high redshift galaxies, I would expect their 100-200 $\mu \mathrm{m}$ luminosity to be comparable or less than that of "normal" spirals at the current epoch in the range $50-100 \mu \mathrm{m}$.

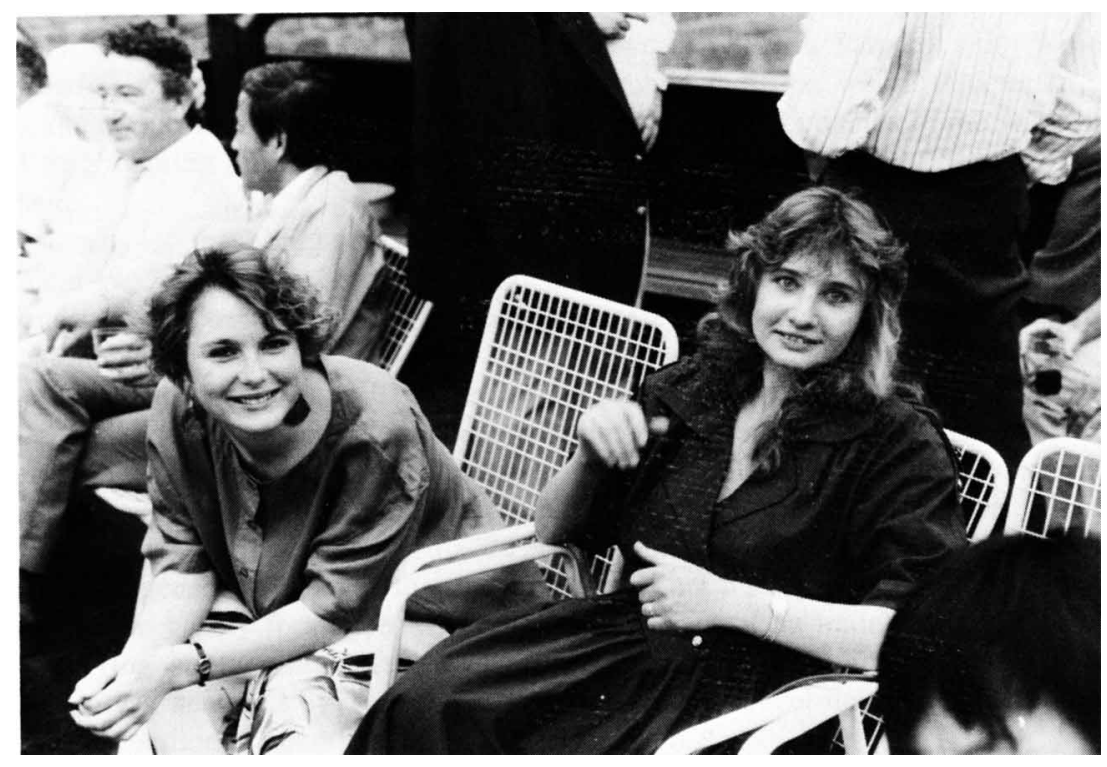

Foreground: Rachel Blythe and Rita Wagner; back left, Giancarlo Setti and Xiang-Tao He 